\title{
Methods for cosmic ray anisotropy searches with AMS-02
}

\author{
Iris Gebauer* \\ Karlsruhe Institute of Technology, Institut für Experimentelle Kernphysik, D-76131 Karlsruhe, \\ Germany \\ E-mail: gebauerekit.edu
}

\section{Karen Andeen; Nikolai Nikonov, Valerio Vagelli; Stefan Zeissler \\ Karlsruhe Institute of Technology, Institut für Experimentelle Kernphysik, D-76131 Karlsruhe, Germany}

The Alpha Magnetic Spectrometer (AMS-02) is a state-of-the-art particle detector designed to operate as an external module on the International Space Station (ISS). In the absence of atmospheric disturbance, cosmic ray fluxes between $0.5 \mathrm{GeV}$ and a few $\mathrm{TeV}$ are measured with high precision. In 2014, the AMS collaboration provided precise measurements of the electron and positron fluxes, which indicate an additional source of positrons among the various cosmic particles. Possible candidates for this source are local pulsars, a local source of positrons produced in proton-gas interactions or the annihilation of dark matter. In the first two cases a possible anisotropy in the electron and positron incoming directions at Earth, caused by the finite extension of the production site, might be detectable.

To determine the level of isotropy in the AMS-02 data it is necessary to compare the measured electron and positron arrival directions to reference maps, which simulate the AMS-02 measurement of an isotropic sky. A common choice of reference maps are proton count maps, assuming that a possible anisotropy in proton arrival directions is significantly lower than that for electrons and positrons.

For protons no reference map can be created from other cosmic ray species: Any anisotropy in cosmic ray arrival directions, caused by the galactic cosmic ray gradient, a local source of secondary or primary cosmic rays, or local structures like the heliosphere, is likely to be present in all cosmic ray species.

We devoloped a method to create a reference map for anisotropy searches, which preserves detector exposure and acceptance, from the arrival directions of the signal map. The method is similar to the shuffling technique with the important difference that it does not preserve the particle counts per sky direction. We argue that our method is better suited to search for anisotropies in cosmic ray arrival directions which exceed the detector's field of view. The method can be applied to all comsic ray species.

The 34th International Cosmic Ray Conference,

30 July- 6 August, 2015

The Hague, The Netherlands

\footnotetext{
* Speaker.

${ }^{\dagger}$ Now at Marquette University, 1250 W. Wisconsin Avenue, Milwaukee, WI 53233, USA.

Now at INFN - Sezione di Perugia, 06123 Perugia, Italy.
} 


\section{Introduction}

In the past years anisotropies in cosmic ray (CR) arrival directions, specifically in the arrival directions of electrons and positrons, have received an increased amount of attention. The PAMELA, Fermi-LAT and AMS-02 [1, 2, 3] experiments have reported on a so-called positron excess, which might be caused by a local point source for electrons an positrons. Indeed, a positive detection of a dipole anisotropy towards a known object (e.g. a pulsar) could prove the astrophysical origin of the anomaly (see e.g. $[4,5]$ and references therein). No detection of any anisotropy has been reported so far, but more and more stringent upper limits are being placed on the total flux of electrons and positrons and on the separate positron flux.

Recently, the AMS-02 collaboration has published the measurement of the proton flux between $1 \mathrm{GV}$ and $1.8 \mathrm{TV}$ [6]. The data show a peculiar change of slope at about $336 \mathrm{GV}$, with a change in spectral index of $\Delta \gamma=0.13$.

The study of proton arrival directions is a crucial ingredient in the understanding of the origin of the spectral features in the proton spectrum. At the same time, limits on the level of anisotropy in proton incoming direction are a necessary cross-check to justify using the incoming directions of protons as a reference map for anisotropy searches in electrons and positrons.

Any search for anisotropies in CR incoming directions requires prior knowledge of the measured distribution of CR arrival directions for a perfectly isotropic sky. The CR incoming directions measured with a detector in the Earth's orbit or on the ground are primarily represented by the detector exposure and acceptance for different regions of the sky.

For anisotropy searches in the incoming directions of electrons and positrons incoming directions, the incoming directions of protons form a viable reference. A possible signal from a local point source of electrons and positrons is not expected in protons, hence a possible anisotropy originating from such a point source might be detectable in a comparison to proton incoming directions.

For protons no such reference from another CR species is available. A possible anisotropy in proton arrival directions may be caused by a variety of circumstances:

- The distribution of supernova remnants peaks at a galactocentric distance of about $4 \mathrm{kpc}$. Protons predominantly escape from our Galaxy via diffusion in the extended galactic halo, but a significant amount of protons from the source region reaches the Sun, leading to a local gradient in the steady state CR distribution and, consequently, a steady flux of protons from the inner galaxy towards the outer galaxy.

- Local structures, such as the local bubble (see, e.g. [8]) or the heliospheric boundary (see e.g. [9]) may induce or wash out anisotropies in the CR arrival directions by virtue of their strong magnetic field turbulences.

- Lastly, local CR accelerators, like close-by supernova remnants, or local regions of increased secondary CR production may imprint information on the production site on CR arrival directions.

The items above are expected to leave a common imprint on all CR species, so that no viable reference map for protons can be deduced from other CR species. Reference maps for protons therefore have to be deduced from the proton data themself. The method usually invoked is the 
so-called shuffling technique [10], which we discuss in section 2. We argue that this method fails to remove anisotropies on scales larger that the detector's field of view. In section 3 we present a novel technique for the creation of reference maps for protons. We apply our method to the AMS-02 data for protons and discuss possible future applications in section 4 . Section 5 concludes our results.

\section{Reference maps}

Here we call a reference map any map which resembles our best estimate of an isotropic sky, measured by the respective detector in the respective data taking period. Any deviation from a reference map may be detected as an anisotropy. Naturally, any reference map needs to preserve the detector's exposure and reproduce the correct detector acceptance. A possible signal needs to be scrambled to a level below the detectors sensitivity.

In 2010 the Fermi collaboration has published upper limits on a possible anisotropy in the combined arrival directions of electrons and positrons above $40 \mathrm{GeV}$ [11]. Two techniques to determine the level of anisptropy were used a) a template fit using a dipole template and b) a significance study of a bin-to-bin comparison. The latter yielded no evidence of an anisotropy in arrival directions and the first method was able to put a limit of $0.5 \%$ for energies larger than 40 $\mathrm{GeV}$ and $10 \%$ for energies larger than $480 \mathrm{GeV}$. Two techniques to create a reference map for electrons were employed, but the majority of the results were derived from the so-called shuffling technique. This method was originally introduced for the search for point sources in UHE cosmic rays with the Fly's eye detector's large field of view [10]. The method is based on the assumption that the detector is responding to an isotropic particle intensity. An artifical dataset is constructed from the actual data set in the following way:

- for each event $i$ taken at time $t_{i}$ with incoming directions in detector coordinates $\left(\phi_{i}, \theta_{i}\right)_{d e t}$, randomly select an event $j$ taken at time $t_{j}$ with incoming directions in detector coordinates $\left(\phi_{j}, \theta_{j}\right)_{d e t}$

- swap the incoming directions of events $i$ and $j$

- calculate the incoming direction in galactic coordinates $\left(\Phi_{i}, \Theta_{i}\right)_{g a l}$ for event $i$ from the incoming directions in detector coordinates $\left(\phi_{j}, \theta_{j}\right)_{\text {det }}$ of event $j$

This proceedure is applied to each event within a selected energy bin. The resulting shuffled map resembles a dataset equivalent to the collected data. The shuffling technique conserves the detectors exposure and acceptance. It is valid for irregular sky coverage even if the overall detector efficiency is highly variable. It only requires stability of the relative rates for different detector directions.

\subsection{Caveats of the shuffling technique}

By construction the shuffling technique described above preserves the particle numbers which are collected from a specific region of the sky. Each event collected in a time period in which the detector's field of view covers a certain region on the sky is replaced with an artificial event originating from the same region of the sky. Anisotropies in CR arrival directions on scales smaller or comparable to the detector field of view will be efficiently washed out by this technique, which makes it 
a suitable approach to search for point sources within the field of view. However, anisotropies on scales much larger than the detector's field of view will be conserved. The Fermi-LAT has a very wide field of view with a useful response to about $60 \mathrm{deg}$ from the instrument zenith axis, covering about $20 \%$ of the sky [12]. The AMS-02 detector has a field of view extending to about $25 \mathrm{deg}$ from the zenith axis for an electron selection and about $35 \mathrm{deg}$ for a proton selection. A possible dipole signal is preserved by the shuffing technique for both of these experiments.

\section{IsoSkyMaps: A novel technique to determine the isotropic sky seen by a certain detector}

To avoid traces of a possible large scale signal in our reference maps we developed two alternative methods for the creation of reference maps for AMS-02. The first method is based on a Monte Carlo estimate of the detector acceptance. This method is described and applied to the AMS-02 electron and positron data in [13]. The second method does not rely on Monte Carlo estimates of the detector acceptance and is described in the following:

A list with proton incoming directions in detector coordinates is created for each rigidity bin. The incoming directions in detector coordinates are then re-assigned by randomly swapping events at different times $t$ and new sky directions are calculated according to the follolwing algorithm:

For each second $t_{i}$ of data taking time:

1. Draw a constant number $N$ of incoming directions in detector coordinates from the list.

2. Recalculate galactic coodinates based on the detector position, orientation and attitude at time $t_{i}$ for $N$ simulated events.

3. Weight events with detector livetime in this second to accont for exposure.

The crucial difference of ths method compared to the shuffling technique is that it does not rely on the single events collected from a certain region of the sky, but instead is based on the livetime of the detector in this region for the respective data taking period.

We verified that the result does not depend on the number $N$ of drawn events by testing the proceedure with $N=5,10,50,100,500,1000$. In additon, separate lists were created for 3 different zones in $\mathrm{GTOD}^{1}$ coordinates. The zones were selected according to the geomagnetic cutoff rigidity $R_{c u t}$ at the respective ISS position, which is calculated based on the International Geomagnetic Reference Field (IGRF) [14] model and are shown in Figs. 1-3: The first zone is defined by $R_{\text {cut }} \leq 6 G V$, the second zone is defined by $6 G V \leq R_{\text {cut }} \leq 15 G V$, and the third zone is defined by $15 G V \leq R_{\text {cut }}$.

The AMS-02 zenith angle is tilted with respect to the ISS zenith by 12 degrees. As a consequence, the AMS-02 detector is pointing in opposite directions for ascending (from magnetic south to magnetic north) and descending (from magnetic north to magnetic sourth) trajectories. We have

\footnotetext{
${ }^{1}$ GTOD: Greenwich True Date Coordinate System. The GTOD system is a rotating, right-handed, Cartesian system with the origin at the center of the Earth. The orientation of this system is specified with: The XY plane is the Earth's true of date Equator, the $\mathrm{Z}$ axis is directed along the Earth's true of date rotational axis and is positive north, the positive $\mathrm{X}$ axis is directed toward the prime meridian, the $\mathrm{Y}$ axis completes a right-handed system.
} 
observed a difference in the proton arrival directions for ascending and descening ISS trajectories, shown in Figs. 4-7. The significances shown in Figs. 6 and 7 are calculated according to the formalism described in [15]. The difference does not exhibit a dependence on particle rigidity, as would be expected from an east-west effect. The origin of this difference is currently under study. To account for the difference in particle arrival directions for ascending and descending ISS trajectories, we subdivide the lists for each zone in two sublists for ascending and descending ISS trajectories. For each second of data taking time the corresponding list is identified and incoming directions in detector coordinates are drawn from the corresponding list.

\subsection{Results}

The resulting IsoSkyMap is shown in Fig. 8 in GTOD and Fig. 9 in galactic coordinates for protons above $40 \mathrm{GV}$ measured with the AMS-02 detector in the period March 2011 to November 2013. A comparison to the proton arrival directions is shown in Fig. 10. The highest significances are located at the South Pole, a region in which the geomagnetic cutoff value and detector livetime is low, and in the region of peculiarities of the Earth's magentic field, like the south atlantic anomaly. This clearly indicates that a finer binning in geomagnetic cutoff is required to correclty take into account the differences in CR arrival directions for different ISS positions. A further systematic may be introduced by asymmetries between the northern and southern hemisphere, as well as the difference in CR arrival directions for ascending and descending ISS trajectories, which might not be fully absorbed in the current analysis. The origin of this effect is currently under study and an update including a full assesment of the systematics of the method will be provided in the future.

\section{Applications for IsoSkyMaps}

IsoSkyMaps were developed primarily for anisotropy searches in protons. The method does not depend on a Monte Carlo based estimate of the detector acceptance and can therefore be employed as a cross check for other methods, as introduced in [13]. In fact, IsoSkyMaps can be used a posteriori to asses the systematics induced by Monte Carlo based acceptance estimates.

Most importantly, the method presented here can be applied to all cosmic ray species. In particular this allows us to search for anisotropies in CR elements heavier than protons. Recently, the AMS colaboration has presented a snapshot of the status of their analyses on helium, lithium and carbon [7]. While the preliminary results shown in these status reports have to be handled with due care, it is noteworthy that both helium and lithium show a hardening in the spectrum, similar to the spectral hardening observed in the published proton spectrum [6]. Possible explanations for this hardening include a change in cosmic ray transport at higher energies, e.g. a break in the diffusion coeffcient, or a new type of source or acceleration mechanism which dominates the local cosmic ray fluxes above a certain energy. Such a source type might imprint a signal upon the arrival directions of cosmic rays.

\section{Conclusion and outlook}

We argued that the shuffling technique preserves a possible large scale anisotropy on scales 


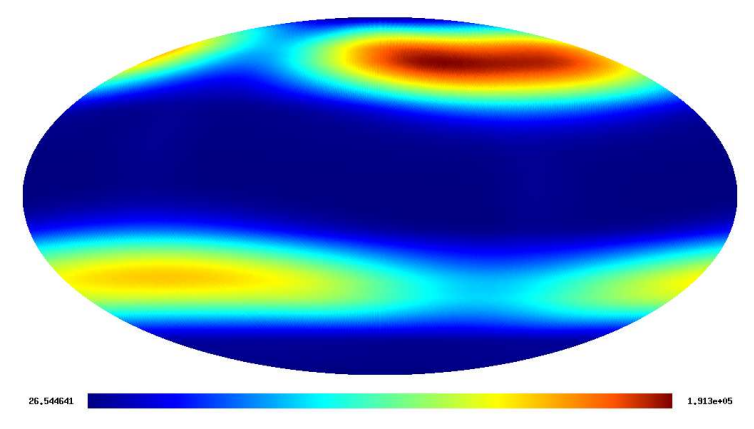

Figure 1: Proton arrival directions for rigidities above $40 \mathrm{GV}$ in GTOD coordinates. Only events with geomagnetic rigidity cutoff $R_{c u t} \leq 6 G V$ are shown.

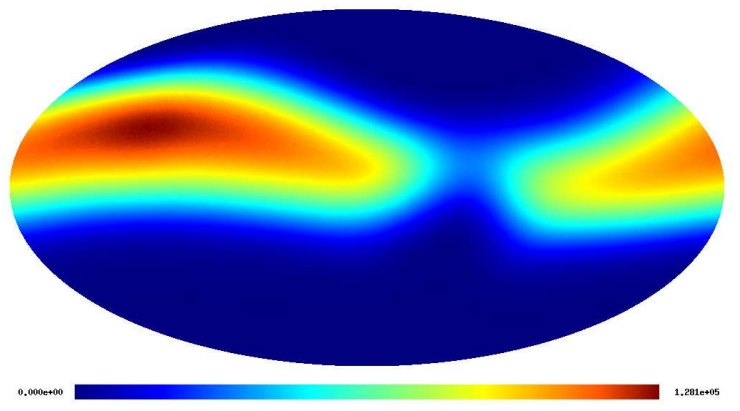

Figure 3: Proton arrival directions for rigidities above $40 \mathrm{GV}$ in GTOD coordinates. Only events with geomagnetic rigidity cutoff $15 G V \leq R_{c u t}$ are shown.

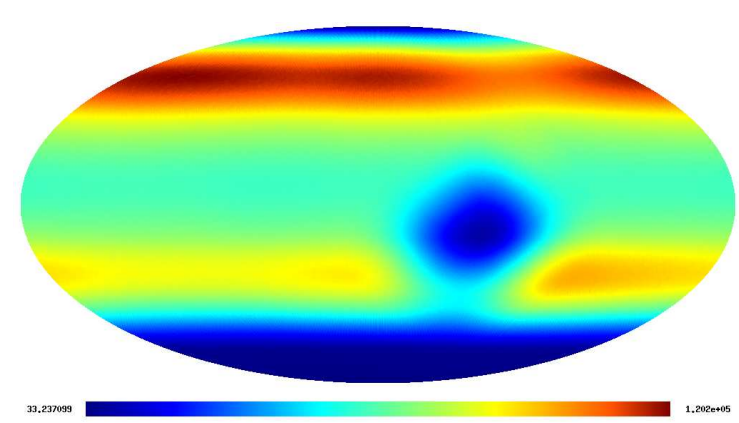

Figure 4: Proton arrival directions for rigidities above $40 \mathrm{GV}$ in GTOD coordinates. Only events collected during times when the ISS is ascending are shown.

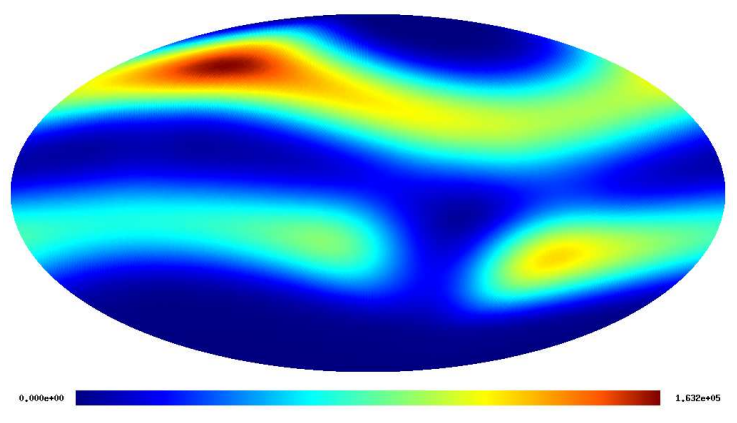

Figure 2: Proton arrival directions for rigidities above $40 \mathrm{GV}$ in GTOD coordinates. Only events with geomagnetic rigidity cutoff $6 G V \leq R_{c u t} \leq$ $15 G V$ are shown.

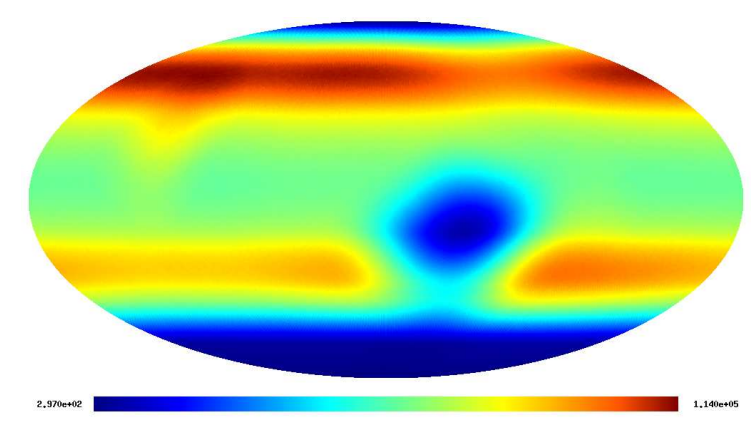

Figure 5: Proton arrival directions for rigidities above $40 \mathrm{GV}$ in GTOD coordinates. Only events collected during times when the ISS is descending are shown. 


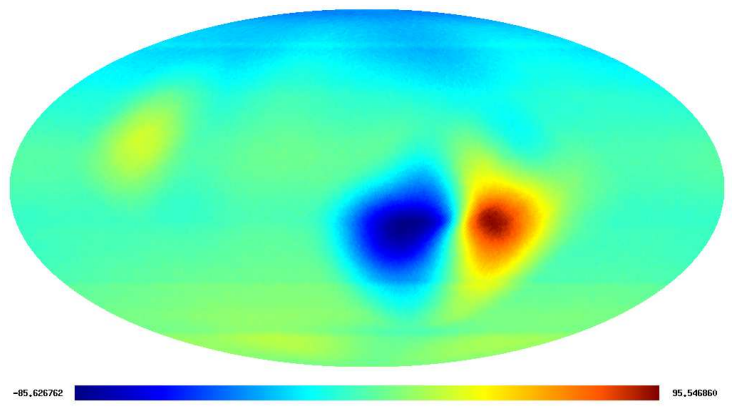

Figure 6: Significance map for the comparison if Figs. 5 and 6. Negative significances are assigned if the counts in the ascending trajectories are smaller than the counts in the descending trajectories. The label of the color scale ranges from -85.63 (dark blue) to 95.55 (dark red).

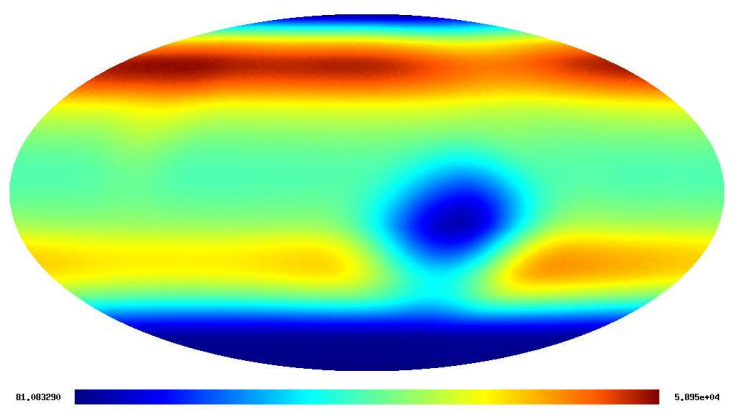

Figure 8: The IsoSkyMap for protons collected with AMS-02 above a rigidity of $40 \mathrm{GV}$ in GTOD coodinates. The increased proton counts towards the Earth's magnetic poles are caused by an increase in exposure due to the ISS orbit. The SAA is clearly visible as a region with low proton counts due to quality selection cuts on the data sample.

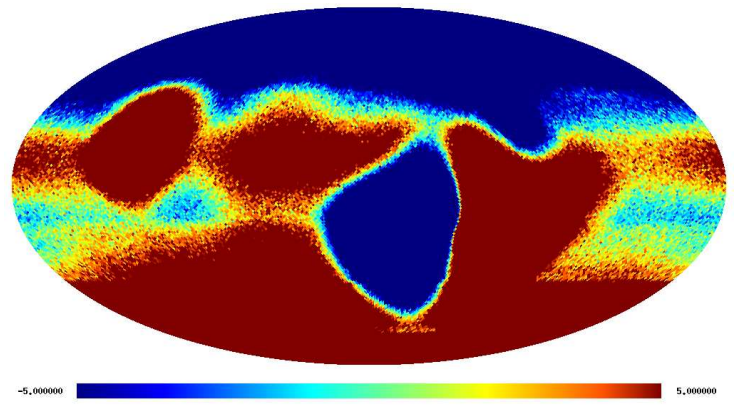

Figure 7: The same as Fig.7, but with the color scale truncated to -5 and 5 .

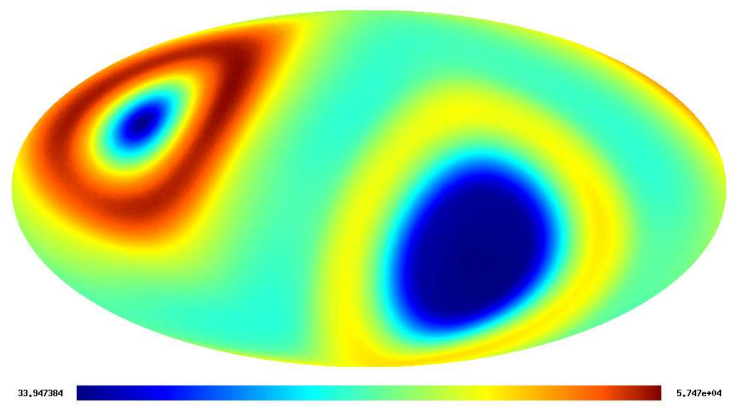

Figure 9: The IsoSkyMap for protons collected with AMS-02 above a rigidity of $40 \mathrm{GV}$ in galactic coodinates. The Earth's North Pole is projected on the circular region with higher proton counts in the upper left part of the figure, the South Pole is in the lower left part of the figure. The SAA is smeared over different regions of the sky.

larger than the detector's field of view. Hence, it is not suitable for dipole anisotropy searches for detectors with a field of view smaller than a hemisphere. For anisotropy searches in electrons and positrons, protons form a suitable reference map, since they can be assumed to be free of the signal of interest. While the local flux of protons is expected to exhibit a small anisotropy due to the fact that most cosmic ray sources are located in the inner galaxy, no significant dipole signal in protons is expected from a pulsar. Maps of proton arrival directions therefore form a viable reference map for anisotropy searches in electrons and positrons, although the systematics due to a possible difference in selections efficiencies and acceptance as a function of geommagnetic position need be taken into account carefully.

For anisotropy searches in the arrival directions of galactic protons no other CR species, which could serve as a basis for a reference map, are available. Here we introduced a novel technique to construct a reference map for protons, based on the arrival directions of protons collected with the 


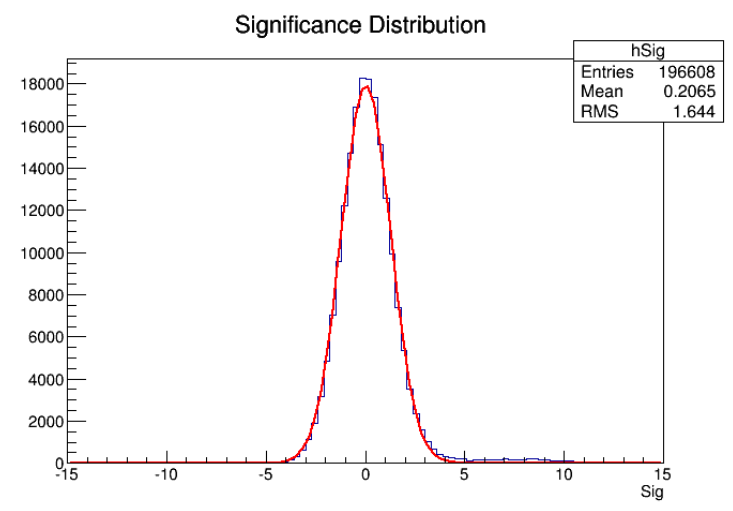

Figure 10: Significance distribution for the comparison between IsoSkyMap and data for rigidities above $40 \mathrm{GV}$. Positive significances are assigned is the counts in the IsoSkyMap exceed the data counts and vice versa. The tail towards positive significances is caused by an excess on the IsoSkyMap towards the South Pole.

respective detector in the respective data taking period. Per construction, our method preserves acceptance and exposure. Different from the shuffling technique it is not based on single events, but instead uses the actual detector livetime for each second of data taking time to simulate the measurement of perfectly isotropic arrival directions. In doing so the method does not preserve a large scale (dipole) anisotropy. The method has been applied to the AMS-02 data on protons as a proof of principle. Future studies are necessary to asses the systematics induced by the choice of the geomagentic cutoff zones and special treatment of regions with low livetime will be required.

The IsoSkyMaps presented here have been created assuming isotropic CR arrival directions at ISS position. Assuming CR arrival directions are isotropic in the insterstellar space, their arrival directions might be distorted by local magnetic fields associated to the heliospheric boundary and the Earth's magnetosphere. For particle transport within the magnetosphere, back-tracing techniques can be applied to single particle trajectories and the measured arrival directions can be projected to the magentospheric boundary. For anisotropy searches in CR arrival directions, an IsoSkyMap for the magentospheric boundary has to be created.

\section{References}

[1] O. Adriani et al. [PAMELA Collaboration], Phys. Rev. Lett. 111, 081102 (2013) [arXiv:1308.0133 [astro-ph.HE]].

[2] M. Ackermann et al. [Fermi-LAT Collaboration], Phys. Rev. Lett. 108, 011103 (2012) [arXiv:1109.0521 [astro-ph.HE]].

[3] L. Accardo et al. [AMS Collaboration], Phys. Rev. Lett. 113, 121101 (2014).

[4] D. Grasso, S. Profumo, A. W. Strong, L. Baldini, R. Bellazzini, E. D. Bloom, J. Bregeon and G. Di Bernardo et al., Nucl. Instrum. Meth. A 630, 48 (2011).

[5] G. Di Bernardo, C. Evoli, D. Gaggero, D. Grasso, L. Maccione and M. N. Mazziotta, Astropart. Phys. 34, 528 (2011) [arXiv:1010.0174 [astro-ph.HE]].

[6] M. Aguilar et al. (AMS Collaboration) Phys. Rev. Lett. 114, 171103 (2015). 
[7] https://indico.cern.ch/event/381134/

[8] I. Gebauer, M. Weinreuter, D. Gaggero, and S. Kunz, The Local Bubble as a cosmic-ray isotropizer, this volume.

[9] N.A.. Schwadron et al. J. Phys. 577, 12023-12033 (2015).

[10] Cassiday, G. L., Cooper, R., Corbató, S. C., et al. 1990, Nuclear Physics B Proceedings Supplements, 14, 291

[11] Ackermann, M., Ajello, M., Atwood, W. B., et al. 2010, Physical Review D, 82, 092003 (2010).

[12] Atwood, W. B., Abdo, A. A., Ackermann, M., et al., APJ, 697, 1071 (2009).

[13] I. Gebauer, K. Andeen, N. Nikonov, V. Vagelli, and S. Zeissler, Direction and time dependent fluxes with AMS-02, this volume.

[14] http://www.ngdc.noaa.gov/IAGA/vmod/igrf.html

[15] Li, T.-P., \& Ma, Y.-Q. APJ 272, 317 (1983) 